\title{
Polyaniline and Mineral Clay-based Conductive Composites
}

\author{
Samantha Oliveira Vilela ${ }^{\mathrm{a}}$, Mauro Alfredo Soto-Oviedo ${ }^{\mathrm{b}}$, Ana Paula Fonseca Albers ${ }^{\mathrm{c}}$, Roselena Faez ${ }^{\mathrm{d} *}$ \\ anstituto de Pesquisa e Desenvolvimento, UNIVAP, \\ Av. Shishima Hifumi, 2911 São José dos Campos - SP, Brazil \\ ${ }^{\mathrm{b}}$ Braskem S.A., Unidade Poliolefinas, Nanotecnologia, Porto Alegre - RS, Brazil \\ ${ }^{\mathrm{c}}$ Geometra BTE, Parque Tecnológico, \\ Av. Shishima Hifumi, 2911 São José dos Campos - SP, Brazil \\ ${ }^{\mathrm{d}}$ Departamento de Ciências Exatas e da Terra, Escola Paulista de Química, \\ Universidade Federal de São Paulo - UNIFESP, 09972-270 Diadema - SP, Brazil
}

Received: March 19, 2007; Revised: August 22, 2007

\begin{abstract}
Composite materials have attracted the attention of numerous researchers due to the distinct properties shown by this class of materials and the mineral clay used in their synthesis. In this study, conductive composites were obtained by mixing polyaniline (PAni) with clay (kaolinite and montmorillonite). The aniline was polymerized in a medium with clay and the powder was characterized by $\mathrm{X}$ ray diffraction, electrical conductivity and morphology. The results suggest PAni chain linearization in a kaolinite medium. The addition of montmorillonite resulted in PAni chain linearization and intercalation of mineral clay, although the montmorillonite was not pretreated. The

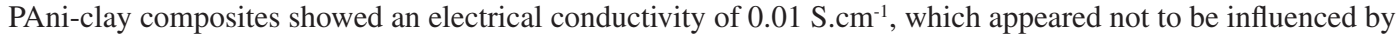
the amount of clay used.
\end{abstract}

Keywords: polyaniline, mineral clay, composites, conducting polymers

\section{Introduction}

Clay materials have been widely employed to obtain composite materials from polymer matrices, particularly due to the fact that these materials can be obtained on a nanometric scale thanks to the lamellar structure of clay minerals, which act as host matrices in the synthesis of the polymer.

Kaolinite clays are abundantly available in nature and are formed by the regular stacking of tetrahedral $\left(\mathrm{SiO}_{4}\right)$ and octahedral $\left(\mathrm{Al}(\mathrm{OH})_{6}\right)$ layers connected to each other in a single layer by the oxygen they share in common. Such layers are stacked regularly and linked by hydrogen bridges, which sufficiently strong provide links to make the cleavage of the material difficult ${ }^{1}$.

Montmorillonite clays are of great industrial interest since they are the clay minerals of predominant use in industry. These clays consist of two tetrahedral silicate $\left(\mathrm{SiO}_{4}\right)$ sheets, with a central octahedral sheet $\left(\mathrm{Al}(\mathrm{OH})_{6}\right)$, connected to each other by the oxygen they share in common. These sheets are stacked one over the other and weakly linked to each other, making it possible for layers of water or polar molecules to accumulate between them ${ }^{1}$.

Montmorillonite is used in the formation and production of nanocomposites because it is easy to insert the polymer material between its layers, although it requires minimization of the surface energy difference between the matrix and the clay. To reduce this difference in surface energy, the clays are organically modified to render them organophilic ${ }^{2,3}$. This treatment also increases the interplanar distance between the lamellae, facilitating the insertion of the monomer and resulting in defect-free polymers with specific properties. In conductive polymers, this chain-wise organization is even more crucial because it raises intra-chain conductivity ${ }^{4,5}$.

The present study involved the production of conductive polyaniline and clay mineral composites without prior organophilization.
The objective of this procedure was to verify the potential for using these raw materials in their natural state.

\section{Materials and Methods}

The kaolinite particles (of Tambaú clay) were separated by sedimentation prior to their use in the synthesis of the composite material. The montmorillonite was not subjected to this procedure because it swells, thus precluding sedimentation.

For the sedimentation process, $20 \mathrm{~g}$ of kaolinite were ground in a mortar and sifted through an ABNT 200 mesh sieve. The resulting powder particles were suspended in a solution of $200 \mathrm{~mL}$ distilled water and $5 \mathrm{~mL}$ ammonium hydroxide and transferred to a $250 \mathrm{~mL}$ test tube. Sedimentation occurred in 24 hours. The supernatant was collected and heated for 24 hours, after which it was ground in a mortar and again sifted through an ABNT 200 mesh sieve.

Ammonium persulfate was added trickle-wise to aniline in a dodecylbenzene sulfonic acid solution (DBSA) to obtain PAni-DBSA. During this procedure, the color of the solution changed visibly, shifting from light to dark green. The sample was filtered, washed and dried to obtain PAni-DBSA powder.

The PAni/clay composite, in the proportions of 10, 20, 30 and $40 \%$ of kaolinite to 10 and $20 \%$ of montmorillonite, was prepared in situ by polymerization of the aniline in DBSA medium. Initially, the solution containing aniline, DBSA and clay was left under constant agitation for 24 hours at $60{ }^{\circ} \mathrm{C}$. The initiator was then added and the procedure continued as described earlier.

The clay, PAni-DBSA and the PAni/clay composite were characterized by X ray diffraction (Shimadzu XRD 6000, $40 \mathrm{kV}$ voltage and $30 \mathrm{~mA}$ current).

Electrical conductivity measurements were taken by the four-point method (Cascade Microtech C4s-64), connected to a source (Keithley 
236), multimeter and amperemeter. The samples were pressed by uniaxial compaction into $\sim 2 \mathrm{~mm}$ thick and $15 \mathrm{~mm}$ diameter compacts. Conductivity was calculated as described in the literature ${ }^{6}$.

The fracture surface of the samples were characterized by scanning electron microscopy (JEOL, model JSM5310).

\section{Results and Discussion}

Figure 1 shows the $\mathrm{X}$ ray diffraction patterns obtained for the PAni produced under different conditions.

The main peaks in the XRD patterns of the PAni-based composites, which were characteristic of the orthorhombic crystalline structure of the emeraldine PAni based polymer, appeared in the positions $(2 \theta)$ of approximately $9.4 ; 15.1 ; 20.4$ and $24.6^{\circ}$.

The PAni-CSA-DCAA XRD pattern ${ }^{7}$ revealed main PAni base peaks slightly displaced, in addition to a peak at $5^{\circ}$, characterizing greater linearization of the polymeric chain ${ }^{7}$, which favors electrical conductivity, as indicated by the results listed in Table 1 .

The main peaks in the PAni-DBSA XRD pattern corresponded to the PAni base; in this case, the peak at $5^{\circ}$ was absent.

Table 1. Electrical conductivity values of the compositions under study.

\begin{tabular}{lc}
\hline \multicolumn{1}{c}{ Composition } & Electrical conductivity $\left({\left.\mathrm{S} . \mathrm{cm}^{-1}\right)}^{-1}\right.$ \\
\hline PAni - emeraldine & $1.0 \times 10^{-9}$ \\
PAni - CSA/DCAA ${ }^{7}$ & $1.1 \times 10^{2}$ \\
PAni - DBSA & 2.5 \\
$10 \%$ Kaolinite & $2 \times 10^{-1}$ \\
$20 \%$ Kaolinite & $5.0 \times 10^{-2}$ \\
$30 \%$ Kaolinite & $3.4 \times 10^{-2}$ \\
$40 \%$ Kaolinite & $2.0 \times 10^{-2}$ \\
$10 \%$ Montmorillonite & $1.1 \times 10^{-1}$ \\
$20 \%$ Montmorillonite & $3.6 \times 10^{-1}$ \\
\hline
\end{tabular}

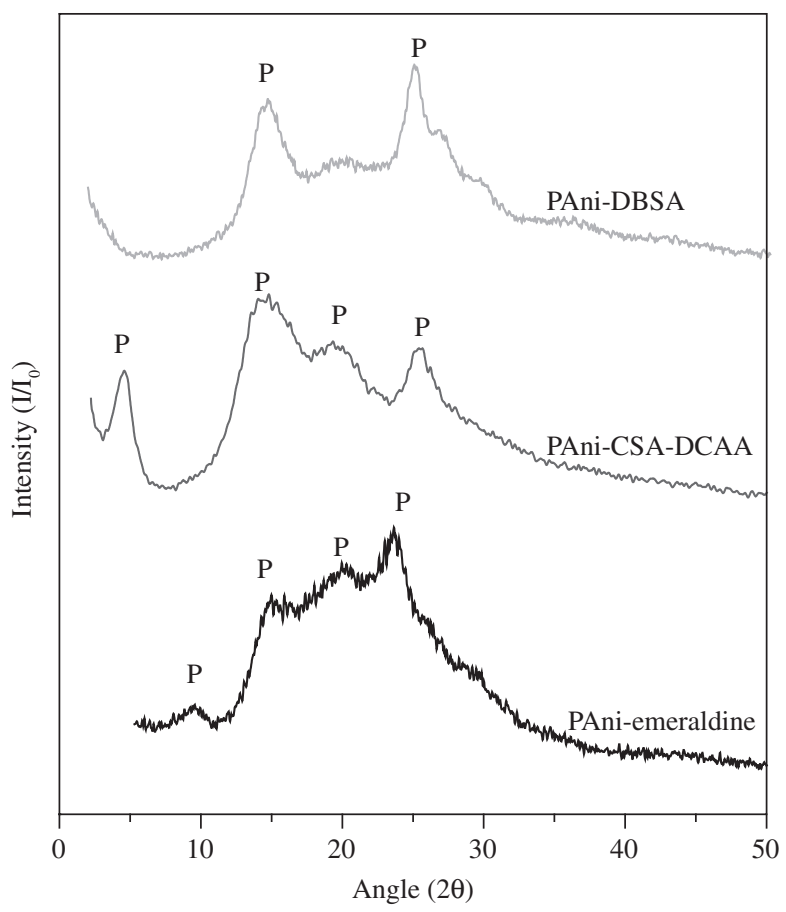

Figure 1. XRD patterns of PAni synthesized under different processing conditions ${ }^{7}$.
Figure 2 presents the XRD patterns obtained for kaolinite and its composites.

Clay XRD pattern presented peaks inherent to the kaolinite crystalline structure in the positions $(2 \theta)$ of $12.6 ; 25.4 ; 35$; and $38^{\circ}$. Peaks corresponding to quartz were also observed at 20 and $26.5^{\circ}$. The purpose of the clay sedimentation process was to eliminate the quartz in the natural raw material. However, it was only partially eliminated, as indicated by the high degree of crystallinity revealed by the peaks in the XRD pattern.

The XRD patterns of the composites containing 10 and $20 \%$ of kaolinite showed not only PAni-based but also kaolinite and quartz peaks.

It is worth mentioning that the addition of mineral clay caused the peak at $5^{\circ}$ to shift to a slightly lower value $\left(2.5^{\circ}\right)$, evidencing greater linearization of the polymeric chain, probably because the electrically negative surface of kaolinite attracts anilinium ions (positive) to its indented surface. This attraction enables the growth of polyaniline on the clay surface, favoring chain linearization. Thus, the kaolinite surface becomes a nucleation site where the polymerization reaction is facilitated.

The electrical conductivity of the composites containing kaolinite was lower than that of the PAni-DBSA (hence, clay is an insulating medium), facilitating the synthesis of these composites and allowing for greater reproducibility. This fact is relevant in the synthesis of conductive polymers, since reproducibility is one of the factors limiting the wider use of this class of materials.

Figure 3 presents the XRD patterns obtained for montmorillonite and its composites containing different amounts of this clay.

The clay's XRD pattern presents the characteristic peak of the montmorillonite crystalline structure in the position $(2 \theta)$ of $7.5^{\circ}$, as well as peaks at 20 and $26.5^{\circ}$, corresponding to the quartz commonly found in clays.

The XRD patterns of the composites containing 10 and $20 \%$ of montmorillonite presented the principal PAni base peaks as well as montmorillonite and quartz peaks. A slightly displacement of the

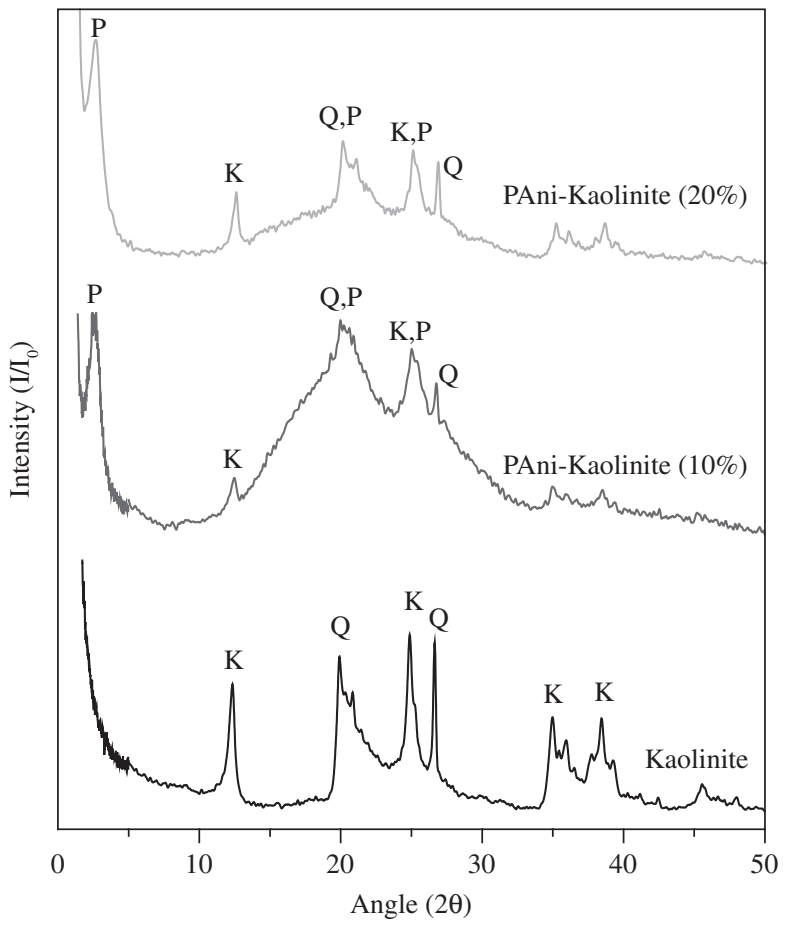

Figure 2. XRD patterns of kaolinite and composites synthesized with different amounts of mineral clay. 


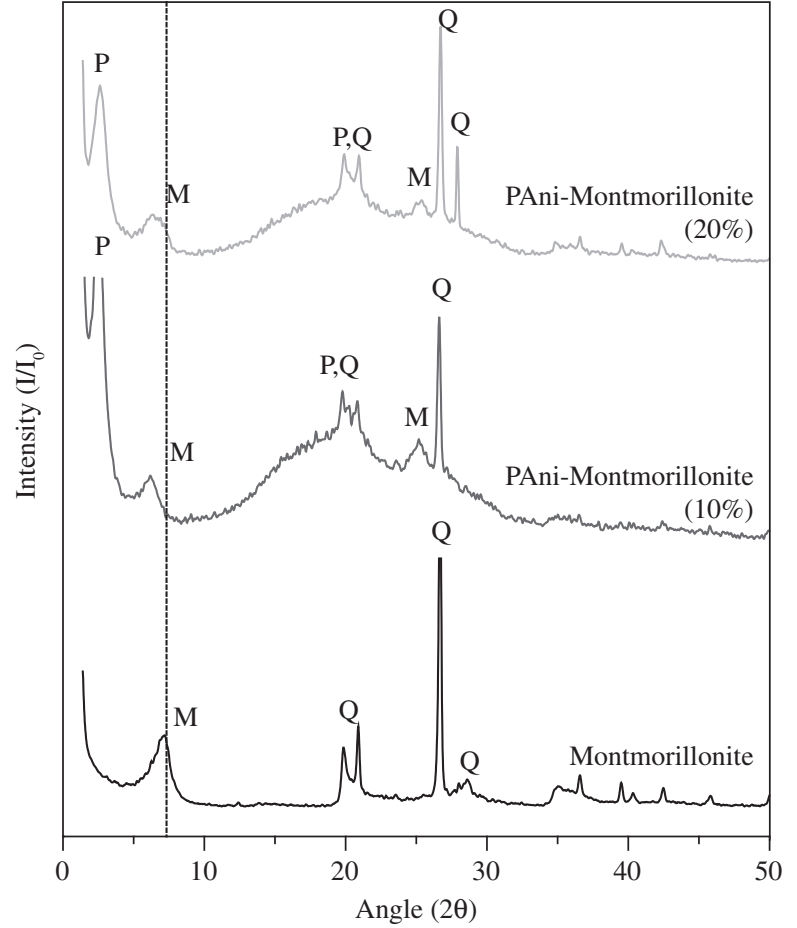

Figure 3. XRD patterns of montmorillonite and composites synthesized with different amounts of mineral clay. peak was also observed at $2.5^{\circ}$, indicating greater linearization of the polymeric chain. It is worth noting that although the montmorillonite was not organophilized, the peak at $7.5^{\circ}$ showed broadening and displacement, characterizing the intercalation of PAni in the montmorillonite ${ }^{8}$. The addition of montmorillonite also facilitated the processing and reproducibility of the synthesis.

Table 1 lists the electrical conductivity results of the composites.

PAni base is an insulator material and the addition of acids to PAni promotes doping, transforming it into a semiconductor. Different types of doping influence the degree of electrical conductivity. The conductivity values for PAni-DBSA were lower than those of PAni-CSA-DCAA, which was attributed to chain linearization, as mentioned earlier herein.

The conductivity values were lower PAni in a clay medium. Regardless of the type of mineral clay employed, samples containing $10 \%$ of clay showed conductivity in the range of $10-1 \mathrm{~S} \mathrm{~cm}-1$; when larger amounts of kaolinite were added, this value decreased by 1 order of magnitude. However, with larger amounts of montmorillonite (up to $20 \%$ ), the conductivity values remained practically constant. This finding suggests that the addition of clay lowers the electrical conductivity, since clay - an insulating material - is added to the medium. However, although montmorillonite is also an insulator, the addition of larger amounts of this clay did not reduce the conductivity, indicating that the electrical conductivity of these samples was favored by an as yet unknown mechanism.

Figure 4 depicts SEM micrographs of PAni-DBSA, PAni-Kaolinite and PAni-Montmorillonite.

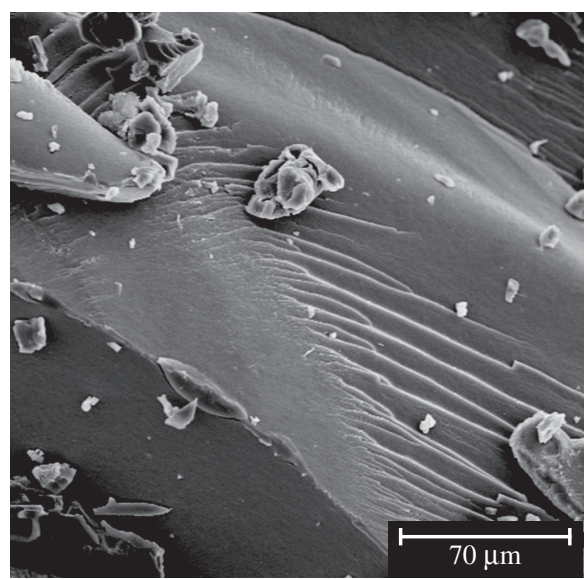

(a)

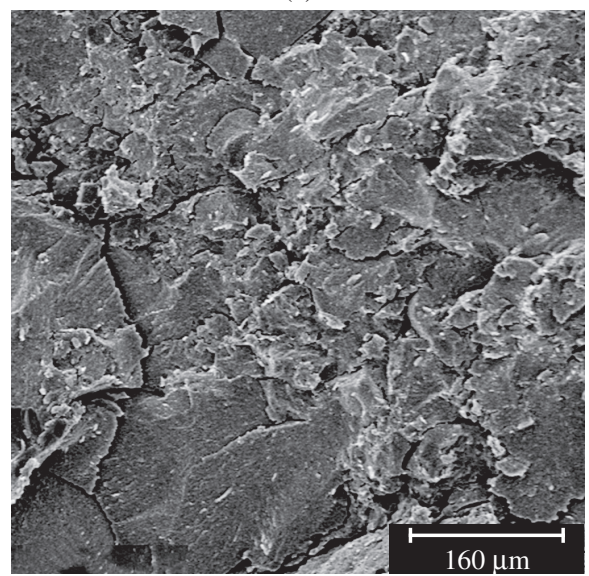

(d)

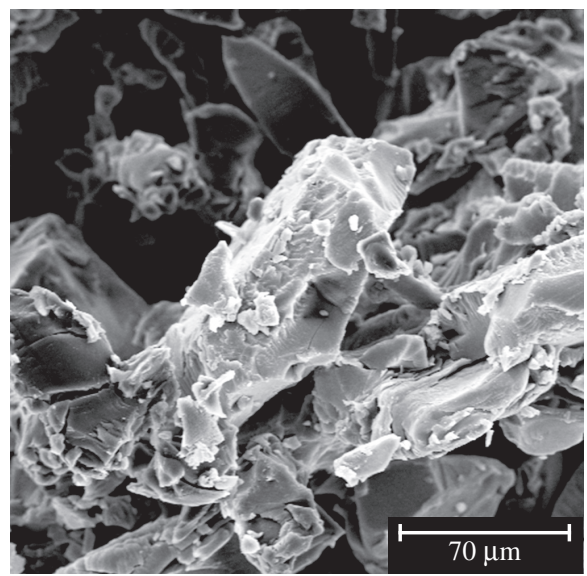

(b)

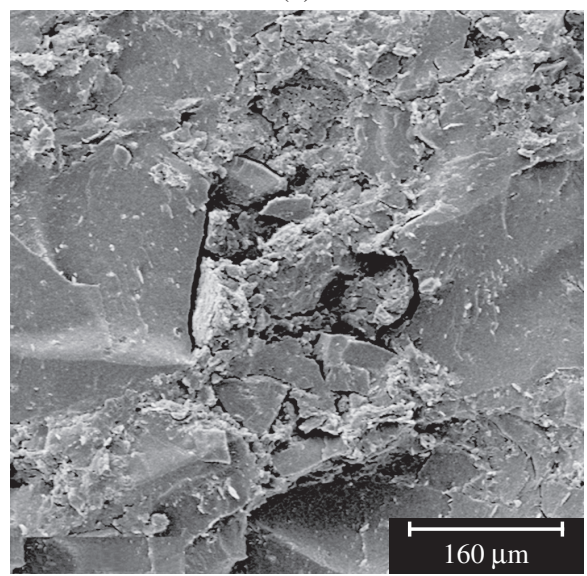

(e)

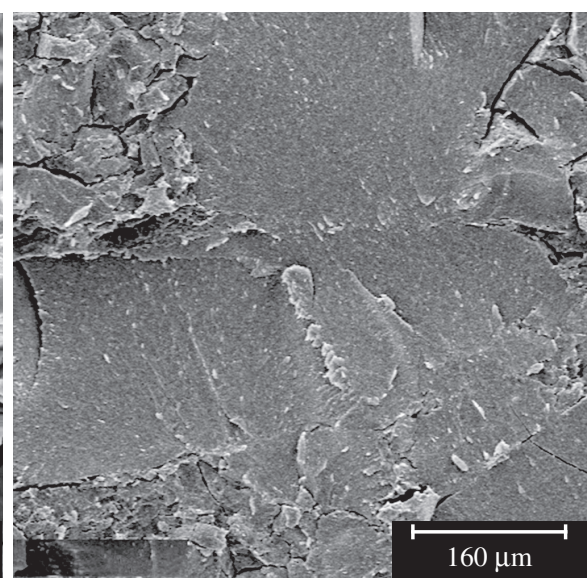

(c)

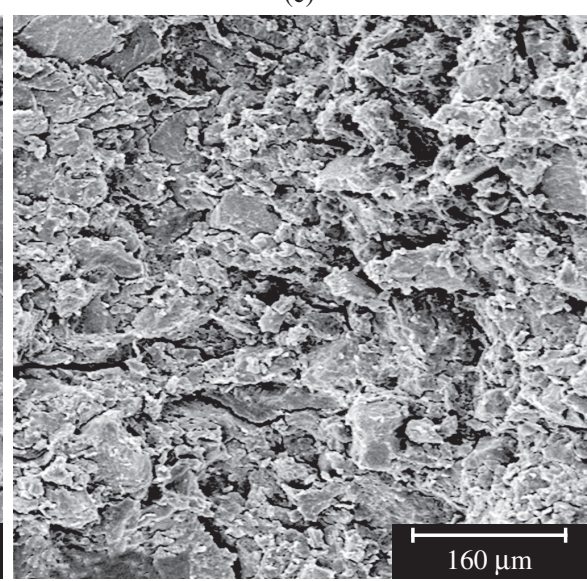

(f)

Figure 4. SEM micrographs of a, b) PAni-DBSA; c) PAni-Kaolinite 10\%; d) 20\%; e) PAni-Montmorillonite $10 \%$; and f) $20 \%$. 
The PAni-DBSA surface fracture is characterized by smooth (a) and rough (b) regions, the former attributed to areas with a higher concentration of doping. The addition of clay to the system resulted in a fracture surface containing more rough regions, which were attributed to the clay, reducing the plastic behavior of the PAni fracture. It is important to point out that the composites containing $20 \%$ of montmorillonite did not show these different regions, presenting, instead, a single rough region characterizing a homogeneous microstructure.

\section{Conclusions}

The XRD patterns suggest linearization of the PAni chain when synthesized in the presence of kaolinite. The PAni-montmorillonite composite presented chain linearization, as well as intercalation of the montmorillonite lamellae, although the montmorillonite was not subjected to organophilization. The addition of clay facilitated the synthesis of PAni and increased the reproducibility of the process. This is a relevant feature in the synthesis of conductive polymers, since one of the limiting factors for the wider use of this class of materials is precisely the reproducibility of the properties obtained.

\section{References}

1. Santos SP. Ciência e tecnologia de argilas. ed. Edgard Blücher LTDA, São Paulo, SP, Brasil; 1989.

2. Kim BH, Jung JH, Kim JW, Choi HJ, Joo J. Effect of dopant and clay on nanocomposites of polyaniline (PAni) intercalated into $\mathrm{Na}^{+}$-montmorillonite (Na+-MMT). Synthetic Metals. 2001; 121(1-3):1311-1312.

3. Wu Q, Xue Z, Qi Z, Wang F. Synthesis and characterization of PAn/clay nanocomposite with extended chain conformation of polyaniline. Polymer. 2000; 41(6):2029-2032.

4. Kim BH, Jung JH, Kim JW, Choi HJ, Joo J. Physical characterization of polyaniline - $\mathrm{Na}^{+}$- montmorillonite nanocompósito intercalated by emulsion polymerization. Synthetic Metals. 2001; 117(1-3):115-118.

5. Kim BH, Jung JH, Hong SH, Kim JW, Choi HJ, Joo J. Physical characterization of emulsion intercalated polyaniline-clay nanocomposite. Current Applied Physics. 2001; 1(1):112-115.

6. Girotto EM, Santos IA. Medidas de resistividade elétrica dc em sólidos: como efetuá-las corretamente. Química Nova. 2002; 25(4):639-647.

7. Biscaro RS, Hoang NH, Wojkiewicz J-L, Faez R, Rezende MC. Blendas de Poliuretano e PAni-CSA Altamente Condutoras e sua Aplicação na Blindagem de Microondas ( $818 \mathrm{Ghz})$. In $8^{\circ}$ Congresso Brasileiro de Polímeros, 2005, Águas de Lindóia, SP.

8. Soto-Oviedo MA, Faez R, Paula AL, Rezende MC, De Paoli M-A. Electromagnetic interference shielding effectiveness of polyaniline-organoclay nanocomposite and EPDM rubber based composites at microwave frequencies, Synthetic Metals. 2006; 156:1249-1255. 